\title{
Bioactive glass S53P4 vs. autologous bone graft for filling defects in patients with chronic osteomyelitis and infected non-unions - a single center experience
}

\author{
Eva Steinhausen ${ }^{1,3}$, Rolf Lefering ${ }^{2}$, Martin Glombitza ${ }^{1}$, Nikolaus Brinkmann ${ }^{1}$, Carsten Vogel ${ }^{3}$, \\ Bastian Mester ${ }^{3}$, and Marcel Dudda ${ }^{1,3}$ \\ ${ }^{1}$ Department of Orthopedic and Trauma Surgery, BG Klinikum Duisburg, \\ University of Duisburg-Essen, 47249 Duisburg, Germany \\ ${ }^{2}$ Institute for Research in Operative Medicine (IFOM), University of Witten/Herdecke, Cologne, Germany \\ ${ }^{3}$ Department of Trauma, Hand and Reconstructive Surgery, University Hospital Essen, \\ University of Duisburg-Essen, Essen, Germany
}

Correspondence: Eva Steinhausen (eva.steinhausen@uni-due.de)

Received: 10 August 2020 - Revised: 9 December 2020 - Accepted: 17 December 2020 - Published: 12 January 2021

\begin{abstract}
Introduction: The goals of osteomyelitis therapy are successful control of infection and reconstruction of the bone. The gold standard for filling defects is the autologous bone graft. Bioactive glass S53P4 is an inorganic bone substitute. We compared the outcome of using bioactive glass (BAG) versus autologous bone graft $(\mathrm{AB})$ in patients with infected non-union. Methods: Patients with chronic osteomyelitis and infected non-union who received either bioactive glass or autologous bone grafts between 2013 and 2017 were analyzed retrospectively. The primary endpoint was successful control of infection during follow-up. Secondary endpoints were bone healing, functional outcome, and occurrence of complications. Results: Eighty-three patients were analyzed (BAG $n=51, \mathrm{AB} n=32$ ). Twenty-one patients experienced reinfection (BAG $n=15,29 \%$; $\mathrm{AB} n=6$, $19 \%$ ). Seventy-eight patients achieved full weight bearing (BAG $n=47,92 \%$; AB $n=31,97 \%$ ). Sixty-four patients had complete bone healing at the end of the follow-up period (BAG $n=39,77 \%$; $\mathrm{AB} n=25,78 \%$ ). There were no significant differences between the groups with respect to the primary or secondary endpoints. Patients with multidrug-resistant pathogens had a significantly higher rate of incomplete bone healing $(p=0.033)$ and a 3-fold higher risk of complications in both groups. Conclusions: Bioactive glass appears to be a suitable bone substitute not only for successful control of infection and defect filling but also for bone healing in cases of infected non-union. In our study, bioactive glass was neither superior nor inferior to autologous bone graft with regard to the primary and secondary endpoints. Further studies with larger numbers of patients are required.
\end{abstract}

\section{Introduction}

Successful infection control is essential in the treatment of chronic osteomyelitis and infected non-union. Adequate surgical debridement remains key to achieving this goal (Ferguson et al., 2017; Lew and Waldvogel, 2004). Surgical debridement should be accompanied by systemic antibiotic treatment. Problems include low antibiotic concentrations due to inadequate perfusion of the bone and the surrounding soft tissues (van Vugt et al., 2016; Romano et al., 2014; Geurts et al., 2011) as well as the development of an- tibiotic resistance and the formation of biofilms (van Gestel et al., 2015; Lindfors et al., 2017). High concentrations can be achieved with local treatment, e.g., with gentamicincontaining polymethylmethacrylate (PMMA) beads. Currently, the gold standard in treating chronic osteomyelitis is a two-stage procedure that involves the use of antibioticcontaining PMMA beads in the first procedural stage (Geurts et al., 2011; Lindfors et al., 2017; van Vugt et al., 2019). However, the PMMA beads must be removed in a further operation (Ferguson et al., 2017; Geurts et al., 2011), and, after releasing the antibiotics, they can themselves act as a 
foreign body, thereby creating a base for bacterial biofilms (Romano et al., 2014; Lindfors et al., 2017; Ferguson et al., 2014; Rahaman et al., 2014).

Bone reconstruction is necessary after successful infection control. Autologous bone graft is the gold standard and has osteogenic, osteoinductive, and osteoconductive properties (Ferguson et al., 2017; Calori et al., 2011; De Long et al., 2007; Egol et al., 2015). However, the volume that can be achieved is limited, and donor site morbidity is considerable (Pape et al., 2010).

Various bone substitutes have been developed in recent years to complement or even replace autologous bone grafting (Egol et al., 2015; Fillingham and Jacobs, 2016; Kurien et al., 2013). An ideal bone substitute should be osteoconductive, osteoinductive, biodegradable, and biocompatible (van Vugt et al., 2016; Calori et al., 2011; Fillingham and Jacobs, 2016). Bone substitutes loaded with antibiotics have been developed for the treatment of bone infections. They usually contain gentamicin (Romano et al., 2014; McNally et al., 2016; Fleiter et al., 2014; Lalidou et al., 2014), or alternatively tobramycin (Ferguson et al., 2014; McKee et al., 2010) or vancomycin (Luo et al., 2016), and make it possible to achieve high local antibiotic concentrations with low systemic levels without incurring the disadvantages of PMMA beads (Ferguson et al., 2017; Lindfors et al., 2017; Ferguson et al., 2014; McNally et al., 2016; Fleiter et al., 2014; McKee et al., 2010; Luo et al., 2016). Clinical evidence in this area is still limited, however (Ferguson et al., 2017; Geurts et al., 2011).

Bioactive glass S53P4 is an inorganic bone substitute with antibacterial, osteoconductive, osteostimulative, and angiogenic properties (Cunha et al., 2018; Coraca-Huber et al., 2014). The results of existing clinical studies of bioactive glass are promising (van Gestel et al., 2015; Lindfors et al., 2017; Ferrando et al., 2017; Lindfors et al., 2010; Auregan and Begue, 2015).

Here, we compare the outcome of bioactive glass S53P4 versus autologous bone grafts for filling defects in patients with chronic osteomyelitis and infected non-union.

\section{Methods}

\subsection{Bioactive glass S53P4 (BAG)}

Bioactive glass (BAG) (Bioglass S53P4; BonAlive ${ }^{\circledR}$ Biomaterials Ltd., Turku, Finland) is an inorganic bone substitute containing $\mathrm{SiO}_{2}, \mathrm{Na}_{2} \mathrm{O}, \mathrm{CaO}$, and $\mathrm{P}_{2} \mathrm{O}_{5}$. Following implantation, sodium and basic ions are released, causing a rapid increase in $\mathrm{pH}$ and osmotic pressure. A silicon dioxiderich layer is formed on the surface. Calcium and phosphate groups migrate to the surface to form a hydroxyl-apatite scaffold. Osteoblasts migrate into this scaffold, proliferate, differentiate, and stimulate osteogenesis. These surface reactions not only stimulate the formation of new bone, but also have an antibacterial effect (Lindfors et al., 2017; Coraca-
Huber et al., 2014; Lindfors et al., 2010). In addition, in vitro studies have shown an angiogenic effect with increased secretion of the vascular endothelial growth factor (VEGF) (Detsch et al., 2014).

\subsection{Patient collective}

We conducted a retrospective analysis of patients treated in our institution for chronic osteomyelitis and infected nonunion who underwent filling of a bone defect with either autologous bone graft or BAG between July 2013 and January 2017.

Inclusion criteria were age $>18$ years, chronic osteomyelitis and infected non-union (positive bacteriology and/or positive histology), filling of the bone defect with either autologous bone graft or BAG, and minimum follow-up of 12 months. Exclusion criteria were aseptic non-unions and lack of follow-up (Fig. 1).

Descriptive epidemiologic data and details of the pre-, peri-, and post-operative courses were compiled from the digital patient files and processed anonymously. Follow-up was analyzed up to a January 2018 cut-off.

The primary endpoint was successful control of infection during follow-up (no clinical, laboratory or radiological signs of infection, closed soft tissues). Secondary endpoints were bone healing and functional outcome focusing on mobilization with full weight bearing. Any complications were recorded.

Bone healing was quantified by evaluating X-ray or CT imaging. Healing was defined as the presence of complete cortical bridging in three out of four cortices or with no evidence of the fracture line.

Multi-resistant pathogens were defined as pathogens resistant to most antibiotics or antibiotic groups (methicillinresistant Staphylococcus aureus, multi-resistant coagulasenegative Staphylococci, multidrug-resistant Gram-negative bacteria and extended spectrum $\beta$-lactamase bacteria).

\subsection{Surgery}

All patients were treated exclusively by the specialized team of the septic surgery department. Decisions regarding treatment method were taken individually for each patient based on surgeon preference.

In all cases, radical debridement was carried out prior to filling the defect. The surgeons took care to completely fill the defect and to press the implanted BAG into the surrounding bone. New samples were taken intraoperatively from all patients.

All patients received antibiotic prophylaxis perioperatively for previously detected pathogens. If an unknown pathogen was present, the patients received cefazoline as the standard antibiotic used in our institution. Patients with an uncomplicated course and sterile samples did not receive postoperative antibiotic treatment. Patients with a pro- 


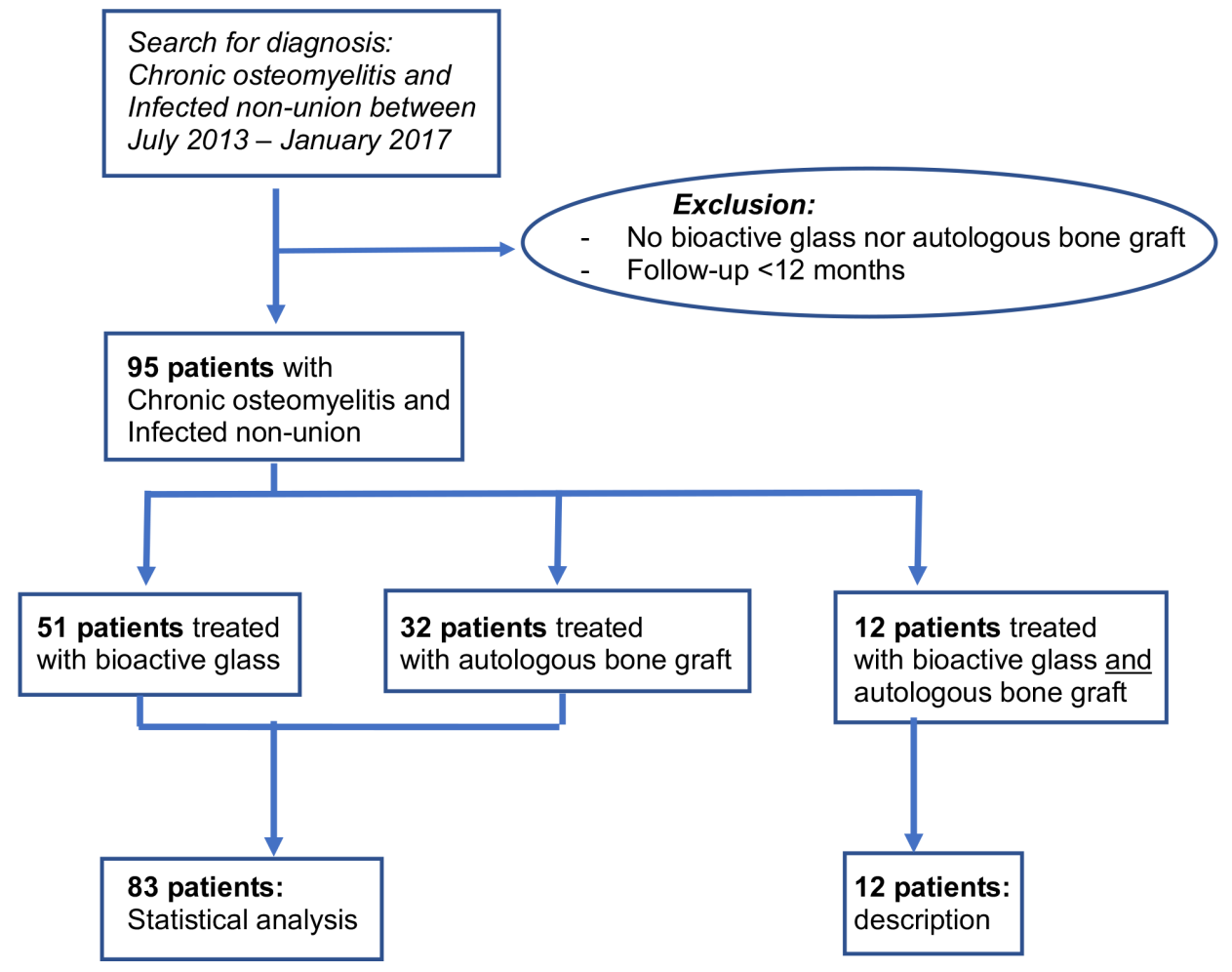

Figure 1. Flowchart of the patient recruitment process.

longed course with multiple previous revisions or with persistent detection of pathogens received postoperative antibiotic treatment. Antibiotic treatment was chosen according to the pathogen and the antibiotic resistance pattern. Antibiotics were given intravenously for 3 weeks and orally for an additional 3 weeks.

\subsection{Statistics}

Treatment groups were compared using Fisher's exact test for binary data and the Mann-Whitney $U$ test for continuous data. A $p$ value $<0.05$ was considered statistically significant. Given the sample size of about 80 cases, the power of this study is limited, especially for even counts. Significant differences for a theoretical prevalence rate of $20 \%$ could be expected only if the prevalence was $<5 \%$ or $>40 \%$ in the other group. For continuous measures, the detectable difference was about half a standard deviation. Statistical analysis was performed using SPSS (Version 24, IBM Inc., Armonk, NY, USA). Multivariate analyses were conducted for complications, recurrence of infection, and osseous fusion.

\section{Results}

Fifty-one patients received bioactive glass S53P4 (BAG), 32 patients autologous bone graft $(\mathrm{AB})$, and 12 patients a combination of bioactive glass and autologous bone graft. The outcomes for these 12 patients are described separately at the end of this section but are not analyzed statistically. Therefore, the following results represent a total of 83 patients (Fig. 1).

\subsection{Preoperative}

The autologous bone graft and the bioactive glass group were comparable with respect to sex, localization and kind of fracture, and the number of previous operations and flaps (Table 1). However, the patients of the BAG group were significantly older and had undergone an unsuccessful prior attempt at defect filling significantly more often than those in the autologous bone graft group. Staphylococcus aureus and coagulase-negative Staphylococci were the most common pathogens involved in both groups. Grampositive pathogens were found significantly more often in the bioactive glass group. Gram-negative pathogens were detected in both groups without significant difference. Multiple pathogens and multidrug-resistant pathogens also did not differ significantly between the groups (Tables 1,2).

\subsection{Perioperative}

In the BAG group, an average of $11 \mathrm{~cm}^{3}$ bioactive glass was implanted (minimum $5 \mathrm{~cm}^{3}$; maximum $30 \mathrm{~cm}^{3}$ ). Intraoperative samples showed persistent bacteria in 17 patients of the $\mathrm{BAG}$ group and in 11 patients of the $\mathrm{AB}$ group, indicating 
Table 1. Epidemiologic data and preoperative findings.

\begin{tabular}{|c|c|c|c|c|}
\hline & Total & $\begin{array}{l}\text { Autologous } \\
\text { bone graft }\end{array}$ & $\begin{array}{r}\text { Bioactive } \\
\text { glass S53P4 }\end{array}$ & $p$ value \\
\hline Total number of patients & 83 & 32 & 51 & \\
\hline Sex (male, \%) & $61(74 \%)$ & $25(78 \%)$ & $36(71 \%)$ & 0.61 \\
\hline Mean age $(y), \mathrm{SD}$ (range) & $52.4 \pm 12.7$ & $45.9 \pm 13.1$ & $56.5 \pm 10.7$ & 0.001 \\
\hline Lower extremity $(n, \%)$ & $78(94 \%)$ & $29(91 \%)$ & $49(96 \%)$ & 0.31 \\
\hline Open fracture $(n, \%)$ & $28(34 \%)$ & $13(41 \%)$ & $15(29 \%)$ & 0.34 \\
\hline No. of previous operations & $21 / 38 / 24$ & $9 / 12 / 11$ & $12 / 26 / 13$ & 0.79 \\
\hline$(<5 / 5-10 />10)$ & $(25 \% / 46 \% / 29 \%)$ & $(28 \% / 38 \% / 34 \%)$ & $(24 \% / 51 \% / 25 \%)$ & \\
\hline Flaps prior to arthrodesis $(n, \%)$ & $30(36 \%)$ & $8(25 \%)$ & $22(43 \%)$ & 0.11 \\
\hline Previous defect filling & $18(22 \%)$ & $2(6 \%)$ & $16(31 \%)$ & 0.007 \\
\hline Multiple pathogens $(n, \%)$ & $34(41 \%)$ & $13(41 \%)$ & $21(41 \%)$ & 0.54 \\
\hline Multidrug-resistant pathogens $(n, \%)$ & $27(33 \%)$ & $14(44 \%)$ & $13(26 \%)$ & 0.097 \\
\hline Gram-positive pathogen $(n, \%)$ & $62(75 \%)$ & $18(56 \%)$ & $44(86 \%)$ & 0.004 \\
\hline Staphylococcus aureus $(n, \%)$ & $30(36 \%)$ & $8(25 \%)$ & $22(43 \%)$ & 0.107 \\
\hline
\end{tabular}

no significant difference between the groups. However, patients of the autologous bone graft group had undergone a change in pathogens significantly more often (BAG $3.9 \%$; AB $28.1 \% ; p=0.001)$. Forty patients of the BAG group and 29 patients of the $\mathrm{AB}$ group received postoperative antibiotic treatment owing to a prolonged course or persistent detection of pathogens. No significant differences were found between the two groups regarding postoperative antibiotic treatment (Table 3).

\subsection{Postoperative}

Patients of the autologous bone graft group had significantly longer follow-up than patients of the bioactive glass group $(p<0.001)$.

Overall, major and minor complications were found in 22 patients of the BAG group and in 20 patients of the AB group, indicating no significant difference between the groups. Recurrence of infection occurred in 15 patients of the BAG group and in 6 patients of the AB group. Statistical analysis also failed to show a significant difference between the groups with regard to recurrence of infection. Two patients $(6.3 \%)$ suffered complications requiring revision surgery after removal of autologous bone from the iliac crest. No complications associated with bioactive glass were observed.

Forty-seven patients of the BAG group and 31 patients of the $\mathrm{AB}$ group achieved full weight bearing during followup, indicating no significant difference in this respect. However, patients of the BAG group achieved full weight bearing significantly more rapidly (BAG 5.9 \pm 4.1 months, median 6 months; AB 10.7 \pm 8.6 months, median 10 months; $p=0.018)$.

The results for bone healing were similar in both groups, with bone healing being seen in 39 patients of the BAG group and in 25 patients of the $\mathrm{AB}$ group by the end of followup. Again, patients in the BAG group accomplished bone healing more rapidly (BAG 9.5 \pm 7.0 months, AB $10.8 \pm 9.0$ months). Eleven patients of the $\mathrm{AB}$ group underwent another procedure to fill the defect, significantly more frequently than in the BAG group (BAG $n=8 ; p=0.049$ ). In addition, patients of the autologous bone graft group underwent reoperations significantly more frequently (BAG $n=24$; AB $n=24 ; p=0.014)$. The rate of below-knee amputation due to recurrent infection during follow-up was comparable between the two groups (BAG $n=3$; $\mathrm{AB} n=2 ; p=1.00$ ) (Table 3$)$.

\subsection{Multivariate analyses}

Detection of multi-resistant pathogens was associated with a significantly higher rate of incomplete osseous fusion ( $p=$ 0.033 ) and was also associated with a 3 -fold increase in the risk of complications (odds ratio 3.091, $95 \%$-confidence interval 1.128-8.470). Male patients had a significantly greater risk of recurrent infection. Finally, there was a 3.5-fold increased risk of recurrent infection after local or free tissue transfer (odds ratio 3.508, 95\%-confidence interval 1.15510.660).

\subsection{Combination of autologous bone graft and bioactive glass}

Twelve patients had a defect filled with a combination of BAG and autologous bone graft. Intraoperative samples revealed persistent bacteria in two patients (16.7\%). Full weight bearing was reached after 4.8 months, on average. Nine patients $(75 \%)$ achieved complete bone healing during follow-up after an average of 7.6 months. Four patients $(33.3 \%)$ developed complications. Only one patient $(8.3 \%)$ had recurrent infection during follow-up. 
Table 2. Causative pathogens identified preoperatively and their proportions.

\begin{tabular}{lrr}
\hline Pathogen & Bioactive glass & Autologous bone graft \\
\hline Total number of patients with known pathogens & $n=45$ & $n=26$ \\
Total number of positive microbiology findings ${ }^{\mathrm{a}}$ & $n=79(100 \%)^{\mathrm{b}}$ & $n=39(100 \%)^{\mathrm{b}}$ \\
Staph. aureus & $n=22(28 \%)$ & $n=8(21 \%)$ \\
COST & $n=22(28 \%)$ & $n=11(28 \%)$ \\
MRSA & $n=2(2 \%)$ & $n=3(7 \%)$ \\
Streptococcus & $n=6(8 \%)$ & $n=0(0 \%)$ \\
Enterococcus & $n=5(6 \%)$ & $n=1(3 \%)$ \\
Enterobacter & $n=3(4 \%)$ & $n=2(5 \%)$ \\
Proteus & $n=1(1 \%)$ & $n=1(3 \%)$ \\
Serratia & $n=3(4 \%)$ & $n=1(3 \%)$ \\
Pseudomonas & $n=6(8 \%)$ & $n=0(0 \%)$ \\
E. coli & $n=3(4 \%)$ & $n=5(12 \%)$ \\
Others & $n=6(8 \%)$ & $n=7(18 \%)$ \\
\hline
\end{tabular}

COST: coagulase-negative Staphylococci; MRSA: methicillin-resistant Staph. aureus; E. coli: Escherichia coli. ${ }^{\text {a }}$ Multiple nominations per patient possible. ${ }^{b}$ Percentages are given in relation to all positive microbiology findings, not in relation to the number of patients; therefore, differences are possible compared to Table 1 (percentages in relation to the number of analyzed patients).

Table 3. Peri- and post-operative findings.

\begin{tabular}{lrrrr}
\hline & $\begin{array}{r}\text { Total } \\
\text { Number of patients }\end{array}$ & $\begin{array}{r}\text { Autologous } \\
\text { bone graft }\end{array}$ & $\begin{array}{r}\text { Bioactive } \\
\text { glass S53P4 }\end{array}$ & $p$ value \\
\hline Perioperative findings & 83 & 32 & 51 \\
\hline Persistent bacteria intraoperative $(n, \%)$ & $28(34 \%)$ & $11(34 \%)$ & $17(33 \%)$ & 1.00 \\
Change in pathogens $(n, \%)$ & $11(13 \%)$ & $9(28 \%)$ & $2(4 \%)$ & 0.001 \\
Postoperative antibiotic treatment $(n, \%)$ & $69(83 \%)$ & $29(91 \%)$ & $40(78 \%)$ & 0.23 \\
\hline Postoperative findings & & & & \\
\hline Follow-up (mean in months; median) & $24.7 \pm 11.8 ; 21$ & $31.3 \pm 12.7 ; 30$ & $20.5 \pm 9.1 ; 18$ & $<0.001$ \\
Recurrence of infection $(n, \%)$ & $21(25 \%)$ & $6(19 \%)$ & $15(29 \%)$ & 0.31 \\
Major and minor complications $(n, \%)$ & $42(51 \%)$ & $20(63 \%)$ & $22(43 \%)$ & 0.12 \\
Bone fusion $(n, \%)$ & $64(77 \%)$ & $25(78 \%)$ & $39(77 \%)$ & 1.00 \\
Full weight bearing $(n, \%)$ & $78(94 \%)$ & $31(97 \%)$ & $47(92 \%)$ & 0.65 \\
Further operations $(n, \%)$ & $48(58 \%)$ & $24(75 \%)$ & $24(47 \%)$ & 0.014 \\
Additional defect filling $(n, \%)$ & $19(23 \%)$ & $11(34 \%)$ & $8(16 \%)$ & 0.049 \\
Amputation $(n, \%)$ & $5(6 \%)$ & $2(6 \%)$ & $3(6 \%)$ & 1.00 \\
\hline
\end{tabular}

\section{Discussion}

The goals of osteomyelitis therapy are successful control of infection and the reconstruction of the bone. Bioactive glass S53P4 is an inorganic bone substitute with antibacterial, osteoconductive, and osteostimulative properties (CoracaHuber et al., 2014; Heikkila et al., 1995).

Our comparison of the outcomes of patients with chronic osteomyelitis and infected non-union who had a defect filled with either autologous bone graft or BAG revealed no significant differences between the groups with respect to recurrence of infection, bone healing, full weight bearing, or complications in general.
Some studies have investigated the use of BAG in patients with chronic osteomyelitis. However, the number of cases in these studies was usually small, and most had no control group (Romano et al., 2014; Lindfors et al., 2017, 2010; Auregan and Begue, 2015; McAndrew et al., 2013; Drago et al., 2013; Malat et al., 2018; Oosthuysen et al., 2020). Existing comparative studies compare BAG either with other bone substitutes (Table 4) or with PMMA beads (the gold standard for a two-stage procedure) and with the objective of investigating successful control of infection and dead space management of bone cavities, but not bone healing. To our knowledge, ours is the first study to compare BAG with au- 
tologous bone graft in patients with an infected non-union that includes fracture healing as a secondary endpoint.

\subsection{Control of infection}

The reinfection rate of patients with chronic osteomyelitis is reportedly as high as $21.2 \%$ (Walenkamp et al., 1998), and the overall reinfection rate after implantation of BAG is $0 \%-$ $14 \%$ according to the current literature (Romano et al., 2014; van Gestel et al., 2015; Lindfors et al., 2017, 2010; McAndrew et al., 2013; Drago et al., 2013; Malat et al., 2018; Tanwar and Ferreira, 2020). We saw higher reinfections rates, especially in the BAG group. However, the number of patients treated with BAG in published studies is usually small. Moreover, the definition of reinfection or persistence of infection used by other authors is less strict than ours. Romano et al. (2014) defined a "fair" outcome as a wound with prolonged drainage or serum leakage of up to 6 weeks and a "poor" outcome as no wound healing for more than 6 weeks or one requiring surgical intervention. Lindfors et al. (2017) defined the outcomes of their patients in a similar way. These findings would be interpreted as persistent infection in our assessment. These differences in definition may explain the increased infection rate in our BAG group. On the other hand, the infection rate of our autologous bone graft group correlates with that reported in other studies. Staphylococcus aureus and coagulase-negative Staphylococci were the most common pathogens involved in both groups. The presence of multi-resistant pathogens was associated with an increased risk of complications regardless of treatment. These results agree with those in other publications. An additional risk factor for recurrent infection in both groups was a previous local or free tissue transfer, as also reported by Lindfors et al. (2017).

All factors considered, we could not establish an advantage of BAG compared to $\mathrm{AB}$ with regard to infection control. There were no significant differences between our two groups in other respects; that is, we found no clear disadvantage for BAG.

\subsection{Bone healing and mobilization with full weight bearing}

Our rate of complete osseous fusion is $77.1 \%$ without significant difference between the groups at latest follow-up. Published studies do not report comparable rates (Romano et al., 2014; van Gestel et al., 2015; Lindfors et al., 2017; Ferrando et al., 2017; Lindfors et al., 2010; McAndrew et al., 2013; Drago et al., 2013). This is unsurprising because these studies investigated the use of BAG only as a bone void filler in chronic osteomyelitis. However, they describe progressive incorporation of BAG, and radiographs therein show partial incorporation at latest follow-up, although the biomaterial remained visible (Romano et al., 2014). In addition, they describe the formation of a thickened cortex (van Gestel et al.,
2015). BAG was not fully incorporated in our patients, either, and we observed the phenomenon of a thickened cortex (Fig. 2).

Ninety-four percent of our patients reached full weight bearing. Thus, we assume that, although osseous fusion remained incomplete on some radiographs, bone consolidation was adequate to ensure stability. Basically, these patients regained a working extremity.

\subsection{Complications}

We observed high complication rates in both groups. The actual rate of reinfection was higher in the BAG group, but patients of the $A B$ group needed significantly more reoperations and significantly more additional defect fillings. Our rate of complications from harvesting autologous bone grafts from the iliac crest is comparable with that reported in other studies (Dimitriou et al., 2011). However, only complications requiring revision surgery are mentioned in our study. Pain after removal of autologous bone graft from the iliac crest and the resulting delay in mobilization are not recorded, although nearly all patients suffer these complications. Last but not least, harvesting an autologous bone graft from the anterior or posterior iliac crest requires prolonged surgery. These drawbacks do not apply to the use of bone substitutes. In our study, bioactive glass was not associated with adverse effects. This matches the experience in the other published studies. So far, there has been no report of BAG-associated complications or formation of bacterial resistance (Romano et al., 2014; van Gestel et al., 2015; Drago et al., 2013). The tolerability of BAG is even described as superior when compared to other bone substitutes (Ferguson et al., 2014; McNally et al., 2016). Van Gestel et al. (2015) referred to bioactive glass $\mathrm{S} 53 \mathrm{P} 4$ as a potential new gold standard.

\subsection{One-stage versus two-stage procedure}

Lindfors et al. (2017) analyzed the largest series of patients $(n=116)$ with chronic osteomyelitis to date, but without a control group. BAG was used in a one-stage procedure in most of the cases, with excellent results. The authors highlight the possibility of one-stage use due to the antibacterial effect as the key advantage of BAG. Other authors agree and underscore its angiogenetic and antibacterial effects (van Gestel et al., 2015; McAndrew et al., 2013; Drago et al., 2013), although radical debridement remains indispensable (Lew and Waldvogel, 2004; Lindfors et al., 2017; Simpson et al., 2001).

Geurts et al. (2011) compared various antibiotic-loaded bone graft substitutes in active or suspected infection with PMMA beads. The authors argue that PMMA beads remain the gold standard even if they require a two-stage procedure. Hence, new biomaterials show great potential, but the level of available evidence is still limited. 
Table 4. Clinical studies investigating the use of bioactive glass S53P4 in patients with chronic osteomyelitis; reviews and animal and in vitro studies are not included.

\begin{tabular}{|c|c|c|c|c|c|}
\hline Author & Year & $\begin{array}{l}\text { No. of } \\
\text { patients }\end{array}$ & $\begin{array}{l}\text { Bone } \\
\text { substitute }\end{array}$ & $\begin{array}{l}\text { Persistent or } \\
\text { reinfection }\end{array}$ & Comment \\
\hline Lindfors & 2017 & 116 & BAG & $12(10.3 \%)$ & No control group \\
\hline Lindfors & 2010 & 11 & BAG & $1(9.1 \%)$ & No control group \\
\hline Drago & 2013 & 27 & BAG & $3(11.1 \%)$ & No control group \\
\hline McAndrew & 2013 & 3 & BAG & $0(0 \%)$ & No control group \\
\hline Romano & 2014 & 76 & $\begin{array}{l}\text { BAG }(n=27) \text { vs. } \\
\text { antibiotic-loaded HA and } \\
\text { calcium sulfate }(n=27) \text { vs. } \\
\text { antibiotic-loaded demineralized } \\
\text { bone matrix and tricalcium } \\
\text { phosphate }(n=22)\end{array}$ & $2(7.4 \%) 3(11.1 \%) 3(13.6 \%)$ & No significant differences \\
\hline Ferrando & 2017 & 25 & $\begin{array}{l}\text { BAG }(n=12) \text { vs. } \\
\text { calcium sulfate antibiotic } \\
\text { beads }(n=13)\end{array}$ & $1(7.7 \%)$ & No significant differences \\
\hline Malat & 2018 & 50 & BAG & $7(14 \%)$ & No control group \\
\hline Oosthuysen & 2019 & 24 & BAG & $2(8 \%)$ & No control group \\
\hline
\end{tabular}

HA: hydroxyapatite; BAG: bioactive glass.

A recently published systematic review has identified a wide range of successful single-stage procedures for the treatment of chronic osteomyelitis including the use of BAG (Pincher et al., 2019). Finally, a recent study has demonstrated the cost-effectiveness of one-stage treatment of chronic osteomyelitis with the bioactive glass S53P4 (Geurts et al., 2019). However, our procedure is different for both BAG and autologous bone graft. We repeated surgical debridement until the intraoperative samples were sterile, in large part because most of our patients had a protracted and therapy-refractory course.

\subsection{Antibacterial effect}

In contrast to autologous bone graft, BAG has antibacterial and angiogenetic effects. These effects are especially advantageous in often poorly vascularized bone. Previous studies show that bacterial biofilms can cause the infecting organism to be resistant to systemic antibiotic concentrations of up to 1000 times greater than normal therapeutic levels (McKee et al., 2010). Such concentrations cannot be achieved with systemic application.

Recently, Ferrando et al. (2017) compared bioactive glass S53P4 $(n=12)$ with calcium sulfate antibiotic beads $(n=$ 13). They did not find significant differences with regard to complication rates and recurrence of infection, but the number of patients was small. The authors conclude that BAG without adjunctive use of local antibiotics is as effective as calcium sulfate antibiotic beads.

Van Vugt et al. (2016) evaluated the use of various bone graft substitutes (Osteoset T, BAG, PerOssal, Herafill-G) in a systematic review ( $n=15$ studies). In general, they criticize weak study design, small number of patients, low levels of evidence (LoE $2 b-3 b)$, and a high risk of bias. Interestingly, the reinfection rate (primary outcome) was higher in high-quality studies. No significant differences were found between the different bone graft substitutes. The authors conclude that the results are inconclusive.

To our knowledge, no study to date compares or proves the superiority of BAG over autologous bone graft with respect to its antibacterial effect.

\subsection{BAG in combination with autologous bone graft}

We combined bioactive glass S53P4 with autologous bone graft in treating large bone defects $(n=12)$ (Fig. 3). The current literature contains no comparable in vivo studies. An in vitro study has analyzed and verified the antibacterial activity of BAG as a bone graft extender in combination with $\mathrm{AB}$ (Bortolin et al., 2018). However, to date there is no evidence to indicate which mixing ratio of bioactive glass and autologous bone graft is optimal. The optimal technique - in layers versus mixed - also remains to be determined. Yet our results in a small number of cases are excellent with respect to our primary and secondary endpoints. Therefore, we prefer the combination of $\mathrm{BAG}$ and $\mathrm{AB}$ for the treatment of large bone defects. However, further evidence in favor of this approach is needed.

\subsection{Limitations}

Our study has some limitations. First, it is a retrospective observational study - although with a control group. The groups are of different sizes, and patients in the BAG group are significantly older. The level of evidence is weak. The maximum follow-up differs between the groups, with signif- 
(a)
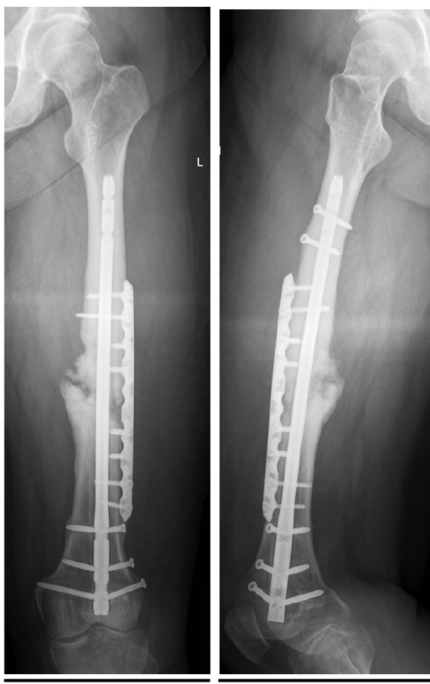

(b)

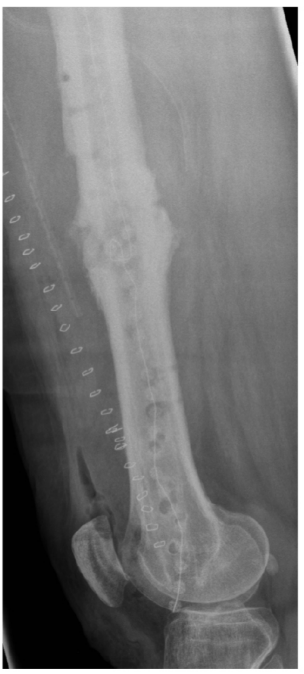

(c)

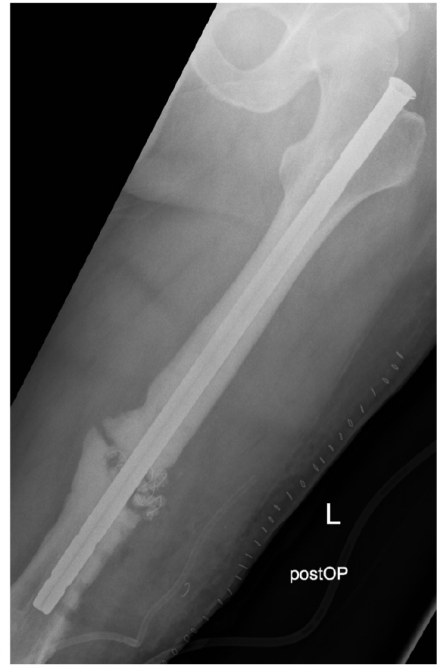

(e)

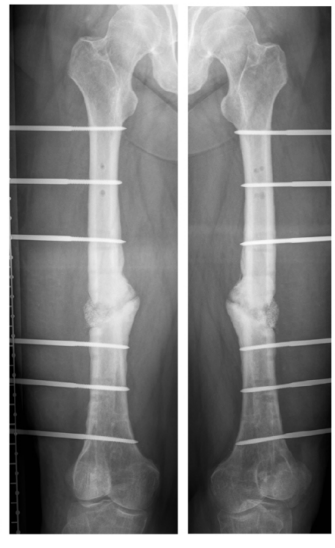

(d)
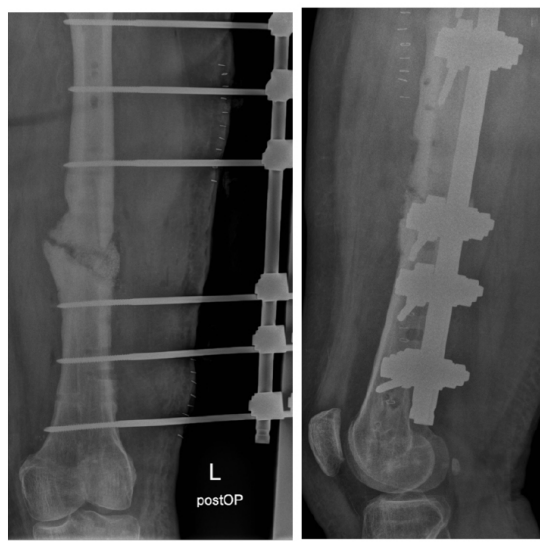

(f)

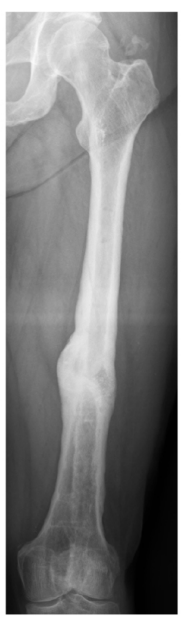

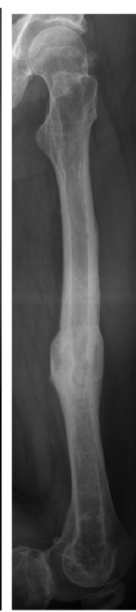

(g)
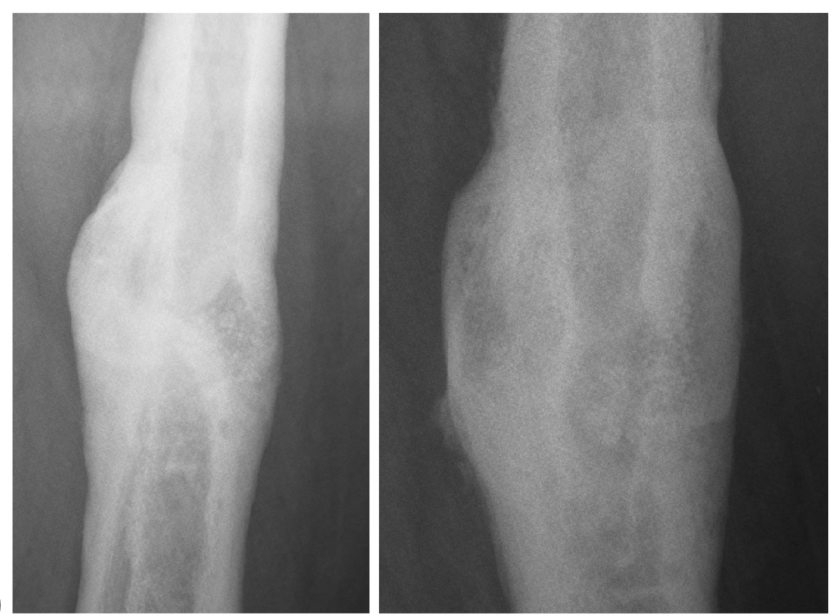

Figure 2. Bioactive glass in a patient with an infected non-union of the femur. (a) Infected non-union of the femur with loosened retrograde intramedullary nail and plate osteosynthesis. (b) After debridement with removal of the internal osteosynthesis, reaming of the intramedullary canal and insertion of a PMMA chain. (c) Temporary stabilization with an antegrade intramedullary nail. (d) Defect filling with bioactive glass after control of infection; definitive stabilization with external fixator. (e) Six months after defect filling. (f) Final result with bony fusion after 2 years. (g) Image enlargement of (f). 
(a)

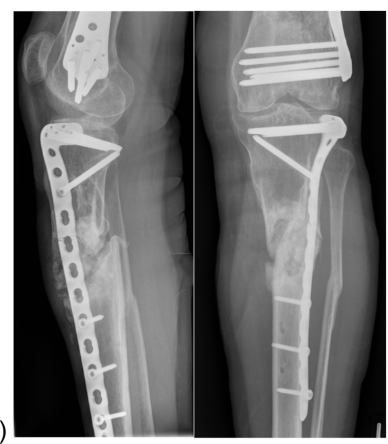

(c)

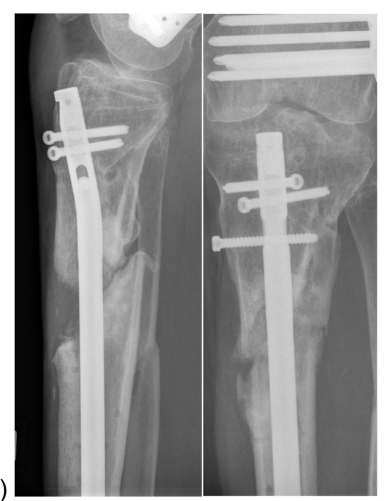

(b)

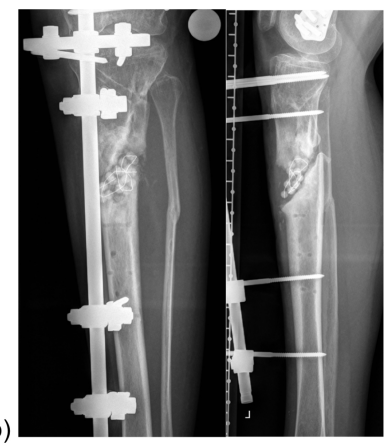

(d)

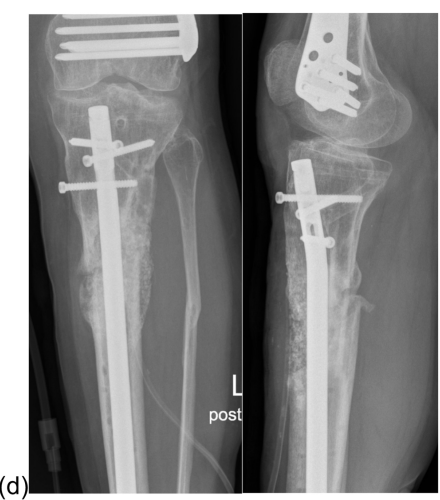

(e)
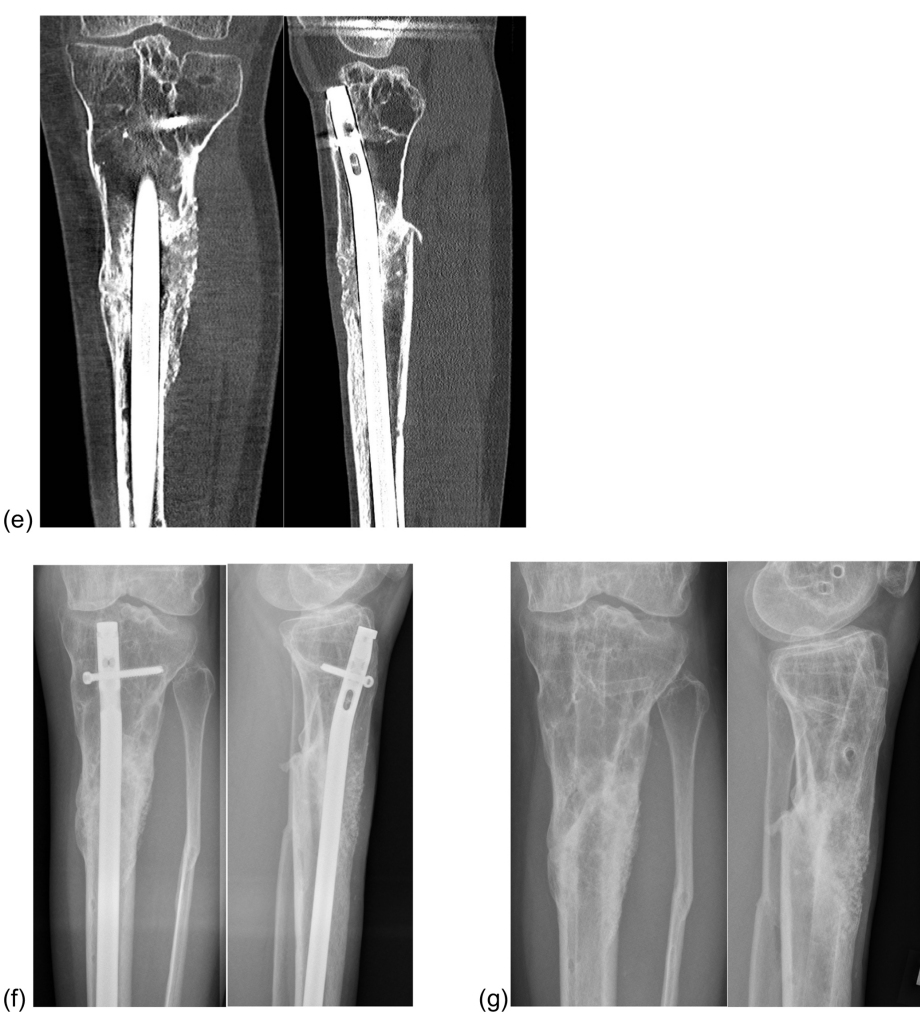

Figure 3. Bioactive glass in combination with autologous bone graft in a patient with an infected non-union of the proximal tibia. (a) Infected non-union of the proximal tibia and plate osteosynthesis. (b) After debridement with removal of the internal osteosynthesis, resection of the infected bone and implantation of a PMMA chain. (c) Re-osteosynthesis with an intramedullary nail after control of infection. (d) Defect filling with a combination of bioactive glass S53P4 and autologous bone graft. (e) Twelve months after defect filling. (f) Two years after defect filling: bony fusion. (g) Final result. Abbreviations: AB: autologous bone graft; BAG: bioactive glass; PMMA: polymethylmethacrylate; VEGF: vascular endothelial growth factor. 
icantly longer follow-up for the $\mathrm{AB}$ group. However, most complications occurred in the 12 months after surgery, so the difference in length of follow-up is not expected to have a significant effect on the observed rate of complications.

\section{Conclusions}

To our knowledge, this is the first study to compare the use of BAG and $\mathrm{AB}$ in patients with chronic osteomyelitis and infected non-union. The results are promising. BAG seems to be an appropriate bone substitute, not only for filling bone defects in patients with chronic osteomyelitis, but also for achieving fracture healing in cases of infected nonunion. In our study, BAG was neither superior nor inferior to autologous bone graft with regard to our primary and secondary endpoints. Prospective randomized studies - as recommended by many authors - would be desirable but are not truly feasible. An analysis with matched pairs may be an alternative.

\section{Ethical statement}

This study was favorably evaluated by the Ethics Committee of Witten/Herdecke University (no. 248/2017).

The study has been performed in accordance with the principles of the Declaration of Helsinki.

We obtained informed consent from the analyzed patients.

Data availability. The datasets analyzed during the current study are available from the corresponding author on reasonable request.

Competing interests. The authors declare that they have no conflict of interest.

Review statement. This paper was edited by Alex McLaren and reviewed by two anonymous referees.

\section{References}

Auregan, J. C. and Begue, T.: Bioactive glass for long bone infection: a systematic review, Injury, 46, Suppl 8, S3-7, https://doi.org/10.1016/S0020-1383(15)30048-6, 2015.

Bortolin, M., Romano, C. L., Bidossi, A., Vecchi, E., Mattina, R., and Drago, L.: BAG-S53P4 as bone graft extender and antimicrobial activity against gentamicin- and vancomycin-resistant bacteria, Future Microbiol., 13, 525-533, https://doi.org/10.2217/fmb2016-0171, 2018.

Calori, G. M., Mazza, E., Colombo, M., and Ripamonti, C.: The use of bone-graft substitutes in large bone defects: any specific needs?, Injury, 42, Suppl 2, S56-63, https://doi.org/10.1016/j.injury.2011.06.011, 2011.
Coraca-Huber, D. C., Fille, M., Hausdorfer, J., Putzer, D., and Nogler, M.: Efficacy of antibacterial bioactive glass S53P4 against $\mathrm{S}$. aureus biofilms grown on titanium discs in vitro, J. Orthop. Res., 32, 175-177, https://doi.org/10.1002/jor.22463, 2014.

Cunha, M. T., Murca, M. A., Nigro, S., Klautau, G. B., and Salles, M. J. C.: In vitro antibacterial activity of bioactive glass S53P4 on multiresistant pathogens causing osteomyelitis and prosthetic joint infection, BMC Infect Dis., 18, 157, https://doi.org/10.1186/s12879-018-3069-x, 2018.

De Long Jr., W. G., Einhorn, T. A., Koval, K., McKee, M., Smith, W., Sanders, R., and Watson, T.: Bone grafts and bone graft substitutes in orthopaedic trauma surgery. A critical analysis, J. Bone Joint Surg. Am., 89, 649-658, https://doi.org/10.2106/JBJS.F.00465, 2007.

Detsch, R., Stoor, P., Grunewald, A., Roether, J. A., Lindfors, N. C., and Boccaccini, A. R.: Increase in VEGF secretion from human fibroblast cells by bioactive glass S53P4 to stimulate angiogenesis in bone, J. Biomed. Mater. Res. A, 102, 4055-4061, https://doi.org/10.1002/jbm.a.35069, 2014.

Dimitriou, R., Mataliotakis, G. I., Angoules, A. G., Kanakaris, N. K., and Giannoudis, P. V.: Complications following autologous bone graft harvesting from the iliac crest and using the RIA: a systematic review, Injury, 42, Suppl 2, S3-15, https://doi.org/10.1016/j.injury.2011.06.015, 2011.

Drago, L., Romano, D., De Vecchi, E., Vassena, C., Logoluso, N., Mattina, R., and Romano, C. L.: Bioactive glass BAG-S53P4 for the adjunctive treatment of chronic osteomyelitis of the long bones: an in vitro and prospective clinical study, BMC Infect. Dis., 13, 584, https://doi.org/10.1186/1471-2334-13-584, 2013.

Egol, K. A., Nauth, A., Lee, M., Pape, H. C., Watson, J. T., and Borrelli Jr., J.: Bone Grafting: Sourcing, Timing, Strategies, and Alternatives, J. Orthop. Trauma., 29, Suppl 12, S10-14, https://doi.org/10.1097/BOT.0000000000000460, 2015.

Ferguson, J., Diefenbeck, M., and McNally, M.: Ceramic Biocomposites as Biodegradable Antibiotic Carriers in the Treatment of Bone Infections, J. Bone Joint Infect., 2, 38-51, https://doi.org/10.7150/jbji.17234, 2017.

Ferguson, J. Y., Dudareva, M., Riley, N. D., Stubbs, D., Atkins, B. L., and McNally, M. A.: The use of a biodegradable antibiotic-loaded calcium sulphate carrier containing tobramycin for the treatment of chronic osteomyelitis: a series of 195 cases, Bone Joint J., 96-B(6), 829-836, https://doi.org/10.1302/0301620X.96B6.32756, 2014.

Ferrando, A., Part, J., and Baeza, J.: Treatment of Cavitary Bone Defects in Chronic Osteomyelitis: Bioactive glass S53P4 vs. Calcium Sulphate Antibiotic Beads, J. Bone Joint Infect., 2, 194201, https://doi.org/10.7150/jbji.20404, 2017.

Fillingham, Y. and Jacobs, J.: Bone grafts and their substitutes, Bone Joint J., 98-B, 1 Suppl A:6-9, https://doi.org/10.1302/0301-620X.98B.36350, 2016.

Fleiter, N., Walter, G., Bosebeck, H., Vogt, S., Buchner, H., Hirschberger, W., and Hoffmann, R.: Clinical use and safety of a novel gentamicin-releasing resorbable bone graft substitute in the treatment of osteomyelitis/osteitis, Bone Joint Res., 3, $223-$ 229, https://doi.org/10.1302/2046-3758.37.2000301, 2014.

Geurts, J., Chris Arts, J. J., and Walenkamp, G. H.: Bone graft substitutes in active or suspected infection. Contra-indicated or not?, Injury, 42, Suppl 2, S82-86, https://doi.org/10.1016/j.injury.2011.06.189, 2011. 
Geurts, J., van Vugt, T., Thijssen, E., and Arts, J. J.: CostEffectiveness Study of One-Stage Treatment of Chronic Osteomyelitis with Bioactive Glass S53P4, Materials (Basel), 12, 3209, https://doi.org/10.3390/ma12193209, 2019.

Heikkila, J. T., Aho, H. J., Yli-Urpo, A., Happonen, R. P., and Aho, A. J.: Bone formation in rabbit cancellous bone defects filled with bioactive glass granules, Acta Orthop. Scand., 66, 463-467, https://doi.org/10.3109/17453679508995588, 1995.

Kurien, T., Pearson, R. G., and Scammell, B. E.: Bone graft substitutes currently available in orthopaedic practice: the evidence for their use, Bone Joint J., 95-B, 583-597, https://doi.org/10.1302/0301-620X.95B5.30286, 2013.

Lalidou, F., Kolios, G., and Drosos, G. I.: Bone infections and bone graft substitutes for local antibiotic therapy, Surg. Technol. Int., 24, 353-362, 2014.

Lew, D. P. and Waldvogel, F. A.: Osteomyelitis, Lancet, 364, 369379, https://doi.org/10.1016/S0140-6736(04)16727-5, 2004.

Lindfors, N., Geurts, J., Drago, L., Arts, J. J., Juutilainen, V., Hyvonen, P., Suda, A. J., Domenico, A., Artiaco, S., Alizadeh, C., Brychcy, A., Bialecki, J., and Romano, C. L.: Antibacterial Bioactive Glass, S53P4, for Chronic Bone Infections A Multinational Study, Adv. Exp. Med. Biol., 971, 81-92, https://doi.org/10.1007/5584_2016_156, 2017.

Lindfors, N. C., Hyvonen, P., Nyyssonen, M., Kirjavainen, M., Kankare, J., Gullichsen, E., and Salo, J.: Bioactive glass S53P4 as bone graft substitute in treatment of osteomyelitis, Bone, 47, 212-218, https://doi.org/10.1016/j.bone.2010.05.030, 2010.

Luo, S., Jiang, T., Yang, Y., Yang, X., and Zhao, J.: Combination therapy with vancomycin-loaded calcium sulfate and vancomycin-loaded PMMA in the treatment of chronic osteomyelitis, BMC Musculoskelet Disord, 17, 502, https://doi.org/10.1186/s12891-016-1352-9, 2016.

Malat, T. A., Glombitza, M., Dahmen, J., Hax, P. M., and Steinhausen, E.: The Use of Bioactive Glass S53P4 as Bone Graft Substitute in the Treatment of Chronic Osteomyelitis and Infected Non-Unions - a Retrospective Study of 50 Patients, Z. Orthop. Unfall, 156, 152-159, https://doi.org/10.1055/s-0043124377, 2018.

McAndrew, J., Efrimescu, C., Sheehan, E., and Niall, D.: Through the looking glass; bioactive glass S53P4 (BonAlive(R)) in the treatment of chronic osteomyelitis, Ir. J. Med. Sci., 182, 509511, https://doi.org/10.1007/s11845-012-0895-5, 2013.

McKee, M. D., Li-Bland, E. A., Wild, L. M., and Schemitsch, E. H.: A prospective, randomized clinical trial comparing an antibiotic-impregnated bioabsorbable bone substitute with standard antibiotic-impregnated cement beads in the treatment of chronic osteomyelitis and infected nonunion, J. Orthop. Trauma, 24, 483-490, https://doi.org/10.1097/BOT.0b013e3181df91d9, 2010.

McNally, M. A., Ferguson, J. Y., Lau, A. C., Diefenbeck, M., Scarborough, M., Ramsden, A. J., and Atkins, B. L.: Singlestage treatment of chronic osteomyelitis with a new absorbable, gentamicin-loaded, calcium sulphate/hydroxyapatite biocomposite: a prospective series of 100 cases, Bone Joint J., 98-B, 12891296, https://doi.org/10.1302/0301-620X.98B9.38057, 2016.
Oosthuysen, W., Venter, R., Tanwar, Y., and Ferreira, N.: Bioactive glass as dead space management following debridement of type 3 chronic osteomyelitis, Int. Orthop., 44, 421-428, https://doi.org/10.1007/s00264-019-04442-7, 2020.

Pape, H. C., Evans, A., and Kobbe, P.: Autologous bone graft: properties and techniques. J Orthop Trauma, 24, Suppl 1, S36-40, https://doi.org/10.1097/BOT.0b013e3181cec4a1, 2010.

Pincher, B., Fenton, C., Jeyapalan, R., Barlow, G., and Sharma, H. K.: A systematic review of the single-stage treatment of chronic osteomyelitis, J. Orthop. Surg. Res., 14, 393, https://doi.org/10.1186/s13018-019-1388-2, 2019.

Rahaman, M. N., Bal, B. S., and Huang, W.: Review: emerging developments in the use of bioactive glasses for treating infected prosthetic joints, Mat. Sci. Eng. C.-Mater., 41, 224-231, https://doi.org/10.1016/j.msec.2014.04.055, 2014.

Romano, C. L., Logoluso, N., Meani, E., Romano, D., De Vecchi, E., Vassena, C., and Drago, L.: A comparative study of the use of bioactive glass S53P4 and antibiotic-loaded calciumbased bone substitutes in the treatment of chronic osteomyelitis: a retrospective comparative study, Bone Joint J., 96-B, 845-850, https://doi.org/10.1302/0301-620X.96B6.33014, 2014.

Simpson, A. H., Deakin, M., and Latham, J. M.: Chronic osteomyelitis. The effect of the extent of surgical resection on infection-free survival, J. Bone Joint Surg. Br., 83, 403-407, https://doi.org/10.1302/0301-620x.83b3.10727, 2001.

Tanwar, Y. S. and Ferreira, N.: The role of bioactive glass in the management of chronic osteomyelitis: a systematic review of literature and current evidence, Infect. Dis. (Lond), 52, 219-226, https://doi.org/10.1080/23744235.2019.1695059, 2020.

van Gestel, N. A., Geurts, J., Hulsen, D. J., van Rietbergen, B., Hofmann, S., and Arts, J. J.: Clinical Applications of S53P4 Bioactive Glass in Bone Healing and Osteomyelitic Treatment: A Literature Review, Biomed. Res. Int., 2015, 684826, https://doi.org/10.1155/2015/684826, 2015.

van Vugt, T. A., Geurts, J., and Arts, J. J.: Clinical Application of Antimicrobial Bone Graft Substitute in Osteomyelitis Treatment: A Systematic Review of Different Bone Graft Substitutes Available in Clinical Treatment of Osteomyelitis, Biomed. Res. Int., 2016, 6984656, https://doi.org/10.1155/2016/6984656, 2016.

van Vugt, T. A. G., Arts, J. J., and Geurts, J. A. P.: Antibiotic-Loaded Polymethylmethacrylate Beads and Spacers in Treatment of Orthopedic Infections and the Role of Biofilm Formation, Front Microbiol., 10, 1626, https://doi.org/10.3389/fmicb.2019.01626, 2019.

Walenkamp, G. H., Kleijn, L. L., and de Leeuw, M.: Osteomyelitis treated with gentamicin-PMMA beads: 100 patients followed for 1-12 years, Acta Orthop. Scand., 69, 518-522, https://doi.org/10.3109/17453679808997790, 1998. 